This item was submitted to Loughborough's Research Repository by the author.

Items in Figshare are protected by copyright, with all rights reserved, unless otherwise indicated.

\title{
Cathode fall characteristics in a dc atmospheric pressure glow discharge
}

PLEASE CITE THE PUBLISHED VERSION

\section{VERSION}

VoR (Version of Record)

LICENCE

CC BY-NC-ND 4.0

\section{REPOSITORY RECORD}

Shi, J.J., and Michael G. Kong. 2019. "Cathode Fall Characteristics in a Dc Atmospheric Pressure Glow Discharge". figshare. https://hdl.handle.net/2134/5170. 
This item was submitted to Loughborough's Institutional Repository (https://dspace.lboro.ac.uk/) by the author and is made available under the following Creative Commons Licence conditions.

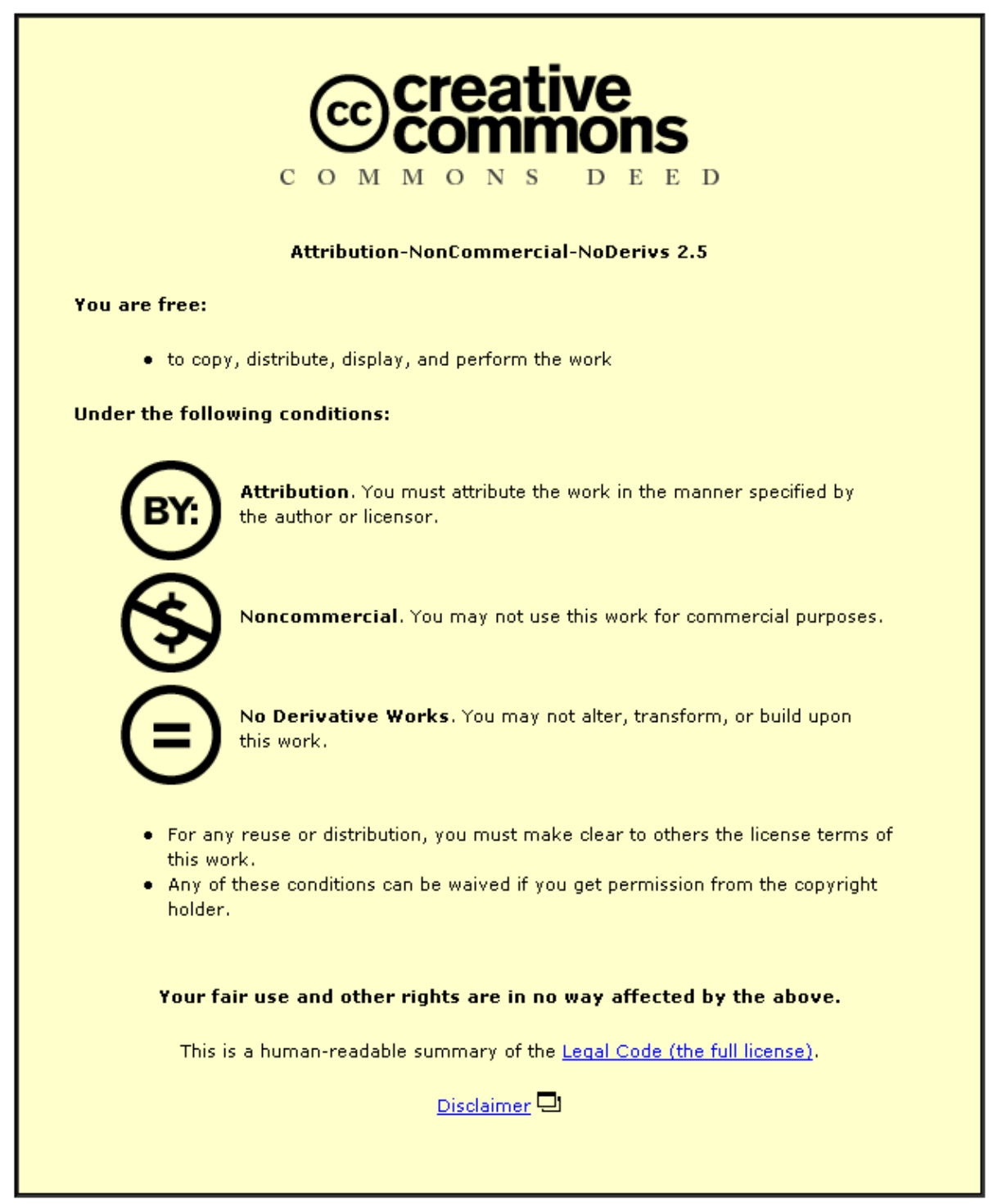

For the full text of this licence, please go to: http://creativecommons.org/licenses/by-nc-nd/2.5/ 


\title{
Cathode fall characteristics in a dc atmospheric pressure glow discharge
}

\author{
J. J. Shi and M. G. Kong ${ }^{\text {a) }}$ \\ Department of Electronic and Electrical Engineering, Loughborough University, \\ Loughborough LE11 3TU, United Kingdom
}

(Received 1 May 2003; accepted 10 August 2003)

\begin{abstract}
Atmospheric pressure glow discharges are attractive for a wide range of material-processing applications largely due to their operation flexibility afforded by removal of the vacuum system. These relatively new atmospheric plasmas are nonequilibrium plasmas with gas temperature around $100{ }^{\circ} \mathrm{C}$ and electron temperature in the $1-10 \mathrm{eV}$ range. Their appearance is characteristically diffuse and uniform, and their temporal features are repetitive and stable. Of the reported numerical studies of atmospheric glow discharges, most are based on the hydrodynamic approximation in which electrons are assumed to be in equilibrium with the local electric field. Spectroscopic and electrical measurements suggest however that the cathode fall region is fundamentally nonequilibrium. To this end we consider a hybrid model that treats the cathode fall region kinetically but retains a hydrodynamic description for the region between the thin cathode fall layer and the anode. Using this hybrid model, a helium discharge system excited at dc is studied numerically for a very wide current density range that spans from Townsend dark discharge, through normal glow discharge, to abnormal glow discharge. Numerical results confirm many distinct characteristics of glow discharges and compare well with that of low-pressure glow discharges. Generic relationships, such as that between the electric field and the current density, are also established and are in good agreement with experimental data. This hybrid model is simple and insightful as a theoretical tool for atmospheric pressure glow discharges. (C) 2003 American Institute of Physics.
\end{abstract}

[DOI: $10.1063 / 1.1615296]$

\section{INTRODUCTION}

There is increasing interest in atmospheric pressure glow discharges (APGDs) largely because they can be used for a wide range of material processing applications without the need for a vacuum system. ${ }^{1-7}$ These nonthermal atmospheric plasmas are typically generated between two parallel electrodes with voltage excitation at $\mathrm{dc},{ }^{8-11}$ at the main frequency, ${ }^{12}$ or at higher frequencies, from kilohertz to megahertz. ${ }^{3-7}$ Their spatial appearance is characteristically diffuse and uniform, and their temporal features are repetitive and stable. Because of their operating at atmospheric pressure, they are highly collisional plasmas with gas temperature around $100{ }^{\circ} \mathrm{C}$ and electron temperature in the $1-10$ $\mathrm{eV}$ range. The main thrust of this field has so far been extensive exploration of their applications, although some of their fundamental properties were characterized experimentally including their discharge dynamics, ${ }^{3-6}$ optical emission, ${ }^{1,13}$ and densities of charged particles and excited species. ${ }^{14,15}$ To a lesser extent they have also been studied numerically often based on hydrodynamics approximation, focusing on discharge dynamics. ${ }^{5,7,16}$ Results of these numerical studies agree favorably with the macroscopic features of measured discharge current and voltage, 5,7 so hydrodynamic models offer useful theoretical tools to understand atmospheric glow discharges. The success of the hydrodynamic models suggests that electrons may be assumed, as an approximation, to be in equilibrium with the local electric field if the modeling

${ }^{a)}$ Electronic mail: m.g.kong@lboro.ac.uk objectives are limited to understanding of the general trend and the macroscopic features of atmospheric pressure glow discharges.

For classical low-pressure glow discharges, it is known that electrons in the usually sub-cm cathode fall region are not in equilibrium with the local electric field. ${ }^{17}$ As the gas pressure is elevated to atmospheric pressure, the muchincreased collision between electrons and neutral particles significantly reduces the thickness of the cathode fall region to around $100 \mu \mathrm{m} .{ }^{9}$ Over this narrow width, secondary electrons released from the cathode are unlikely to gain sufficient acceleration and reach equilibrium with the local electric field. Therefore the hydrodynamic models typically used for nonthermal atmospheric plasmas are in principle inappropriate for the cathode fall region of the discharge even though such models have been successful in predicting the general trend of some discharge properties. Given that accurate modeling of the cathode fall region is essential for realistic prediction of electron density and electron energy that decisively influences plasma chemistry and hence intended applications, it is highly desirable to develop an improved model for atmospheric glow discharges taking into account the nonequilibrium nature of their cathode fall region. In this article, we consider a hybrid model that treats the cathode fall region using a kinetic technique and retains the hydrodynamic description for the remaining regions of discharge. These two treatments are integrated at the near-anode boundary of the cathode fall region as shown in Fig. 1 such that the current density and electric field transit the interface smoothly. It is worth mentioning that the cathode fall struc- 


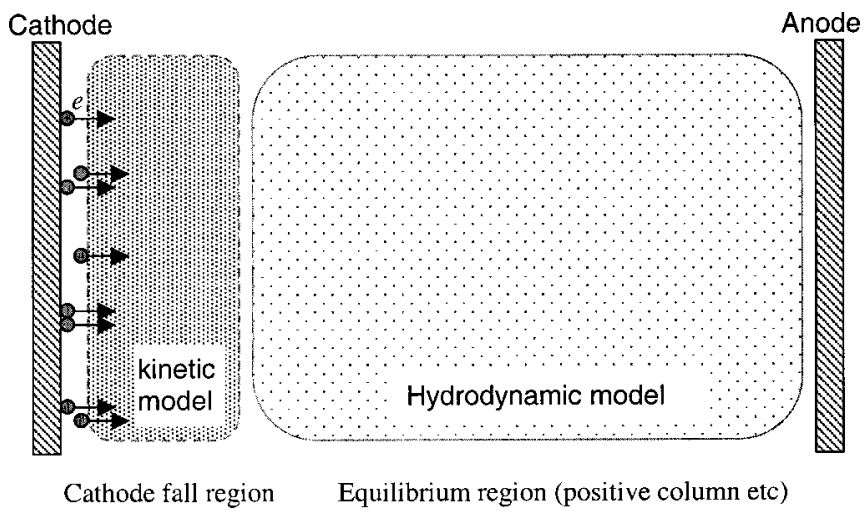

FIG. 1. Schematic of a hybrid model for a capacitively coupled dc plasma system.

ture can be alternatively treated with a full kinetic model such as those used for low-pressure glow discharges. ${ }^{18}$ Such an approach often relies on Monte Carlo simulations and in general demands much greater computational resources. ${ }^{18}$ The proposed hybrid model is simpler, more intuitive, and capable of unraveling the majority of key cathode fall features, as will become clear in Secs. II-IV. Furthermore, this simple model and its findings will also form a good basis on which to develop and refine full kinetic treatment of APGD that has been scarce in the literature. ${ }^{19}$

In principle the proposed hybrid model can be developed for any type of APGD, and in this article it will be developed for APGD generated by dc excitation. This is motivated partly by the obvious practicality of dc plasmas and partly by a substantial amount of experimental studies of dc APGD. ${ }^{8-12,20-30}$ It is conceivable that the proposed hybrid model can in principle be amended readily for application to ac excited atmospheric pressure glow discharges. As the first step and favoring its generic application, our development of the hybrid model will be based on a monatomic noble gas system to which plasma chemistry can be added at a later stage for application to specific gas-mixture systems. The article will be organized as follows. In Sec. II we will develop the physical basis and the mathematical framework of the proposed hybrid model based on a similar hybrid model that has been applied successfully to low-pressure glow discharges. By removing the hydrodynamic assumption, electron impact ionization near the cathode is determined from the mean electron energy rather than from the local electric field thus enabling kinetic treatment of the cathode fall region. The hydrodynamic description is retained for the space between the cathode fall region and the anode (e.g., Faraday dark space, the positive column, and anode dark space). In Sec. III, the hybrid model will be employed to unravel structural characteristics of typical dc atmospheric discharge through numerical computation of the spatial variation of key physical quantities including the electric field, the electron current density, the mean electron energy, and the electron and ion densities. The results confirm the distinct features of glow discharges and compare well qualitatively with those of low-pressure glow plasmas. In Sec. IV several important relationships will be explored, for example, that between the electric field and the current density. Similarities to and contrast from those of low-pressure glow discharges will be studied and discussed. Finally in Sec. V some concluding remarks will be summarized.

\section{HYBRID MODEL AND ITS BASIC EQUATIONS}

We consider a capacitively coupled plasma system consisting of two parallel flat metallic plates across which a dc voltage, $V_{g}$, is applied. As an example of monatomic gas systems we consider atmospheric discharges in pure helium. Suppose the gas plasma starts as a Townsend discharge. This occurs when the gap voltage exceeds the breakdown voltage of the monatomic gas and the Townsend breakdown condition applies, ${ }^{17}$

$$
\Delta \equiv \gamma\left(e^{\alpha_{0} L}-1\right)-1,
$$

where $\alpha_{0}$ and $\gamma$ are the first and the second Townsend coefficients when the gap voltage is equal to the breakdown voltage. $L$ is the gap between the two electrodes. The discharge current grows when $\Delta$ is positive and decreases when $\Delta$ is negative. According to Ward, ${ }^{31}$ the first Townsend coefficient in helium discharges is given by

$$
\alpha=A p \exp \left[-B(p / E)^{1 / 2}\right],
$$

where $p$ is the gas pressure, $E$ is the local electric field at which $\alpha$ is assessed, $A=6.5 /$ (cm Torr), and $B$ $=16.4 \mathrm{~V}^{1 / 2} /\left(\mathrm{cm}^{1 / 2} \operatorname{Torr}^{1 / 2}\right)$. The use of the above formula implicitly assumes that electrons are in equilibrium with the local electric field and gas ionization can be viewed as completely determinable from the local field. For nonthermal atmospheric helium discharges, spectroscopic measurements suggest that the electric field at the cathode surface is as high as $45 \mathrm{kV} / \mathrm{cm}$. ${ }^{9}$ The use of such a large value of local electric field in Eq. (2) would lead to a gross overestimate of the ionization level at the cathode surface. Realistically, secondary electrons are released from the cathode with kinetic energy typically in the $0.5-1 \mathrm{eV}$ range $\mathrm{e}^{17,32,33}$ and at this energy level they are rather inefficient in impacting substantial ionization. They need to be accelerated considerably in the cathode fall region before reaching equilibrium with the local electric field.

One simple technique by which to account for the nonequilibrium cathode fall is to relate the Townsend ionization coefficient to the mean electron energy, $\bar{\varepsilon}$, rather than to the local electric field. More specifically, for the cathode fall region the Townsend first coefficient is modified to ${ }^{32,33}$

$$
\alpha=A p \exp \left[-B^{\prime}(1 / \bar{\varepsilon})^{1 / 2}\right],
$$

where $B^{\prime}=16.4 \mathrm{~V}^{1 / 2}$. This relationship is established for low-pressure glow discharges from their Monte Carlo based kinetic simulations, ${ }^{18}$ and its application to low-pressure glow discharges has been successful in reliably predicting the cathode fall characteristics. ${ }^{32,33}$ Since Eq. (3) is concerned with the fundamental processes of electron impact ionization in the cathode fall region of a glow discharge, it should be independent of the gas pressure and also applicable to atmospheric glow plasmas, particularly given spectroscopic and electrical observations that the cathode fall characteristics of atmospheric glow plasmas are very similar to those of low-pressure glow plasmas. ${ }^{5,934}$ Therefore we 
assume that Eq. (3) is applicable at atmospheric pressure although currently there are no reported Monte Carlo simulations of APGD similar to those performed for low-pressure glow discharges ${ }^{18}$ that can be used to evaluate this assumption directly. The assumed applicability of Eq. (3) to APGD will be assessed and confirmed in Secs. III and IV by comparison with experiments of numerical predictions resulting from this assumption.

With the ionization coefficient evaluated from Eq. (3), the cathode fall region is effectively treated kinetically. For the remaining part of the discharge (e.g., the Faraday dark space, the positive column, and the anode dark space ${ }^{5,17,34}$ ), we assume that electrons there are in equilibrium with the local electric field ${ }^{17,33}$ and their impact ionization can be described by Eq. (2). Thus effectively we now model the cathode fall region kinetically and the remaining discharge region hydrodynamically. This is in essence identical to a similar hybrid approach used successfully for low-pressure glow discharges. ${ }^{32,33}$ Again, similar to the treatment for lowpressure glow discharges, ${ }^{32,33}$ we assume that the ion diffusion coefficients and electron and ion mobility can be approximated by those evaluated under hydrodynamic conditions throughout the discharge. The validity of this approximation will be assessed and confirmed in Secs. III and IV by comparison with experiments of numerical predictions of the proposed hybrid model.

If the electrode gap is much smaller than that characterizing the electrode surface area, multidimensional effects are less important and negligible. Thus the governing equations of discharge dynamics of a monatomic gas are given as ${ }^{7,17}$

$$
\begin{aligned}
& \frac{\partial n_{e}}{\partial t}+\frac{\partial\left(n_{e} v_{e}\right)}{\partial z}=\alpha n_{e} v_{e}-R n_{e} n_{i}, \\
& \frac{\partial n_{i}}{\partial t}+\frac{\partial\left(n_{i} v_{i}\right)}{\partial z}=\alpha n_{e} v_{e}-R n_{e} n_{i}, \\
& \frac{\partial(\varepsilon E)}{\partial z}=|e|\left(n_{i}-n_{e}\right), \\
& J_{i, e}= \pm|e| n_{i, e} v_{i, e}, \\
& v_{i, e}= \pm \mu_{i, e} E-\left(D_{i, e} / n_{i, e}\right) \partial n_{i, e} / \partial z .
\end{aligned}
$$

Here $n_{e}(z)$ and $n_{i}(z)$ are the electron and ion densities, and $J_{i, e}$ and $v_{i, e}$ are their current densities and drift velocities. $\mu_{i, e}$ and $D_{i, e}$ are their mobilities and diffusion coefficients. $R$ is the recombination coefficient. For simplicity, excited helium species and impurities such as nitrogen are not considered in the above equations so stepwise and Penning ionization $^{5,7}$ are excluded. Hence we anticipate numerical results of Eq. (4) to indicate an ionization level that is lower than that observed experimentally. ${ }^{7}$ Under dc excitation voltage and assuming that the discharge reaches its steady state without arcing, the time dependence of all physical quantities is ignored. Equation (4e) assumes that electrons and ions reach their equilibrium drift velocities on a time and space scale that is much shorter than that characterizing the changing electric field. Under dc excitation and at atmospheric pressure these are easily satisfied.
Equations (4) can be further simplified by the fact that the recombination terms in Eqs. (4a) and (4b) are usually negligible..$^{32,33}$ The recombination rate is about 2 $\times 10^{-12} \mathrm{~cm}^{3} / \mathrm{s}$ for $\mathrm{He}^{+}$and the ion density is up to $10^{13} \mathrm{~cm}^{-3}$, whereas even at a low electric field of $3 \mathrm{kV} / \mathrm{cm}$ the ionization coefficient is about $1.3 \mathrm{~cm}^{-1}$ and the electron mobility is $1132 \mathrm{~cm}^{2} /(\mathrm{V} \mathrm{s}) .{ }^{17}$ These numbers suggest that the recombination term is much smaller than the ionization term and as such is ignored. Therefore

$$
\begin{aligned}
\frac{d J_{e}}{d z} & =\alpha J_{e}, \\
\frac{d J_{i}}{d z} & =-\alpha J_{e}, \\
\frac{d E}{d z} & =-\frac{1}{\varepsilon_{0} \varepsilon_{r}}\left[\frac{J_{i}}{v_{i}}-\frac{J_{e}}{v_{e}}\right] \\
& =-\frac{J_{i}+J_{e}}{\varepsilon_{0} \varepsilon_{r} v_{i}}\left[1-\frac{J_{e}}{J_{i}+J_{e}}\left(1+\frac{v_{i}}{v_{e}}\right)\right] .
\end{aligned}
$$

The first two equations suggest that the total current density $J=J_{i}+J_{e}$ remains constant during discharge. We denote $j_{e}$ $=J_{e} / J$ as the normalized electron current density to reduce the above equations to

$$
\begin{aligned}
& \frac{d j_{e}}{d z}=\alpha j_{e}, \\
& \frac{d E}{d z}=-\frac{J}{\varepsilon_{0} \varepsilon_{r} v_{i}}\left[1-\left(1+\frac{v_{i}}{v_{e}}\right) j_{e}(z)\right] .
\end{aligned}
$$

The electron drift velocity is related to the electron mobility and the diffusion coefficient in Eq. (4e). With a stationary background gas (e.g., not one in an external flow), the diffusion term contributes much less than the mobility term to the electron drift velocity. ${ }^{32}$ Thus the electron drift velocity can be approximated as

$$
v_{e}=\mu_{e} E,
$$

where $\mu_{e} p=0.86 \times 10^{6} \mathrm{~cm}^{2}$ Torr/(V s) for helium. ${ }^{17}$ On the other hand, the ion drift velocity is given by the Ward formula, ${ }^{31}$

$$
v_{i}=\left\{\begin{array}{cl}
\mu_{i}(E / p)[1-C(E / p)] & E / p \leqslant W_{1}, \\
k_{i} \sqrt{E / p}\left[1-D\left((p / E)^{3 / 2}\right)\right] & E / p>W_{1} .
\end{array}\right.
$$

Here $W_{1}=25 \mathrm{~V} /(\mathrm{cm}$ Torr $), \mu_{i}=8 \times 10^{3} \mathrm{~cm}^{2}$ Torr $/(\mathrm{V} \mathrm{s}), k_{i}$ $=4.1 \times 10^{4} \mathrm{~cm}^{3 / 2} \mathrm{Torr}^{1 / 2} /\left(\mathrm{V}^{1 / 2} \mathrm{~s}\right), \quad C=8 \times 10^{-3}(\mathrm{~cm} \mathrm{Torr}) /$ $\mathrm{V}$, and $D=27.44(\mathrm{~V} / \mathrm{cm} / \text { Torr })^{3 / 2}$.

In the proposed hybrid model, its kinetic treatment of the cathode fall region and its hydrodynamic description for the remaining discharge are integrated via a weighting function ${ }^{33}$

$$
W=\frac{\exp \left[\left(z-d_{c}\right) / d_{1}\right]}{1+\exp \left[\left(z-d_{c}\right) / d_{1}\right]},
$$

where $d_{c}$ is the thickness of the cathode fall region and $d_{1}$ is an empirically deduced length-controlling parameter. ${ }^{33}$ Typically we choose $d_{1}=0.2 d_{c}$ as in the case for low-pressure glow discharges ${ }^{33}$ although numerical simulations for both low-pressure and atmospheric pressure glow discharges sug- 
gest that the choice of $d_{1}$ is not crucial to the basic features of $j_{e}, E$, and $\varepsilon$. This will be confirmed in Sec. III. The weighting function of Eq. (9) is small near the cathode surface $(z=0)$ and increases exponentially towards unity beyond the near-anode boundary of the cathode fall $\left(z=d_{c}\right)$. It is used to determine effective electron energy, ${ }^{33}$

$$
\varepsilon_{1}=W(E / p)+(1-W) \kappa \bar{\varepsilon},
$$

where $\kappa=1 /($ Torr $\mathrm{cm})$. Equation (10) is then used in the following universal ionization formula: ${ }^{33}$

$$
\alpha=A p \exp \left[-B\left(1 / \varepsilon_{1}\right)^{1 / 2}\right] .
$$

The mean electron energy is determined from the energy flux equation $^{18,33}$

$$
\frac{d \bar{\varepsilon}}{d z}=E(z)-\left(\bar{\varepsilon}+U_{i}\right) \alpha-U_{e} \delta_{e},
$$

where $\delta_{e}$ is the coefficient for the production of excited helium states, $U_{e}=21.45 \mathrm{eV}$ is the onset potential for excited states, and $U_{i}=24.58 \mathrm{eV}$ is the onset potential for ionized states, all of helium. A comparison of cross sections for excitation and ionization in helium as a function of electron energy suggests that $\delta_{e}=0.5 \alpha$ is a reasonable approximation. ${ }^{18,33}$ Numerical results suggest that deviation from this simplified relationship matters little for the spatial dependence of the electric field and the current density. Again this will be confirmed in Sec. III.

Equations (6a), (6b), and (12) provide a complete set of equations for three unknowns, namely, the electric field, the electron current density, and the mean electron energy, thus they form the working equations for the helium discharge system. The boundary conditions are (a) the mean electron energy is $1 \mathrm{eV}$ at the cathode; ${ }^{18,33}$ (b) the initial normalized electron current density is $j_{e}(0)=\gamma /(1+\gamma)$ at the cathode and $j_{e}(L)=1$ at the anode. Before simulation, the cathode fall thickness $d_{c}$ is assumed and the cathode electric field $E_{c}$ is selected numerically such that the electric field at the nearanode boundary of the cathode fall region $\left(z=d_{c}\right)$ is a small preset fraction of $E_{c}$. In all numerical examples presented here, this small fraction is chosen as $14 \%$. If the initial choice of $E_{c}$ is too large or too small, numerical solution of the working equations [Eqs. (6a), (6b), and (12)] suggests that either $j_{e}$ becomes greater than unity or $E$ become negative. These two scenarios are unlikely to be physical and as such are omitted. In practice, numerical computations of the working equations confirm that once $j_{e}(0), d_{c}$, and $J$ are chosen, the cathode electric field is uniquely determined by the working equations.

The use of the spatial location of $0.14 E_{c}$ to determine $d_{c}$ is based on the fact that reduction of the electric field from the cathode surface is largely linear from $E_{c}$ to $0.14 E_{c}$. If a smaller ending point is used, say, $0.1 E_{c}, E$ may undergo nonlinear reduction between 0.14 and $0.1 E_{c}$ for the numerical examples considered. Because the extent of the nonlinear $E$ reduction differs in different cases, we choose the $14 \%$ rule to remove the differential effect of nonlinear field reduction. Also, although $d_{c}$ determined with the $0.14 E_{c}$ rule differs from that determined with a $0.1 E_{c}$ rule, their difference is small and is found to influence little the conclusions to be drawn from this work.

\section{STRUCTURAL CHARACTERISTICS OF dc APGD}

To unravel the typical structure of dc excited atmospheric glow discharges, we consider a helium plasma with total current density $J=0.18 \mathrm{~A} / \mathrm{cm}^{2}$, normalized cathode electron current density $j_{e}(0)=0.2(\gamma=0.25)$, electrode gap $L=0.4 \mathrm{~cm}$, and assumed cathode fall thickness $d_{c}$ $=65.5 \mu \mathrm{m}$. The choice of $\gamma=0.25$ is based on data for platinum in helium, ${ }^{17}$ and is useful to aid in a comparison of the numerical results with experimental data in Sec. IV. Numerical computation of the working equations [e.g., Eqs. (6) and (12)] suggests that the cathode electric field needs to be within $\pm 1.3 \times 10^{-4} \%$ of $39.90275 \mathrm{kV} / \mathrm{cm}$ in order to yield physically meaningful results. A greater cathode electric field would lead to $j_{e}(z)$ going above unity thus requiring direction reversal of the ion current density $\left[j_{i}(z)=1-j_{e}(z)\right.$ $<0$ ], whereas a smaller cathode electric field would reverse the direction of the electric field at some point within the cathode fall region. These scenarios are unlikely to lead to the usual structure of glow discharges ${ }^{5,17,18,30-34}$ even if there may be plausible conditions for them to be possible. So we will not consider these scenarios further. The extreme narrowness of the cathode electric field range $\left( \pm 1.3 \times 10^{-4} \%\right)$ suggests that the APGD system as defined by the working equations is a self-regulating one capable of yielding an effectively unique cathode electric field in response to a preselected set of $J$ and $j_{e}(0)$. This should be compared to the unique relationship between the electric field and the total current density commonly observed in low-pressure glow discharges. ${ }^{17}$ Consequently for all numerical examples discussed here in Sec. III, we will use a fixed cathode electric field at $39.90275 \mathrm{kV} / \mathrm{cm}$.

Figures 2(a)-2(f) show the spatial dependence of the electric field, the normalized electron current density, the mean electron energy, the ionization coefficient, the electron density, and the ion density, respectively. Densities of charged particles are calculated from Eq. (4d) using the electron and ion current densities. In the cathode fall region, it is clear that the electric field decreases almost linearly from the cathode surface and this spatial variation is very similar to that observed in low-pressure glow discharges. ${ }^{17,32,33}$ This also agrees with the field profile measured spectroscopically for a helium dc atmospheric glow discharge. ${ }^{9}$ While the electric field reduces over the width of the cathode fall region, both the electron energy [Fig. 2(c)] and the ionization coefficient [shown by the solid curve in Fig. 2(d)] increase. The dotted curve in Fig. 2(d) is the ionization coefficient calculated from Eq. (2) using the electric field in Fig. 2(a) and it highlights the overestimation of the ionization level near the cathode surface by the hydrodynamic model. From Fig. 2(c), on the other hand, it is clear that the mean electron energy increases rapidly from $1 \mathrm{eV}$ at the cathode surface and reaches its maximum of $39 \mathrm{eV}$ around $40 \mu \mathrm{m}$ [see the inset in Fig. 2(c)] before gradually tailing off. The spatial variation of the mean electron energy in Fig. 2(c) mirrors that of ion- 

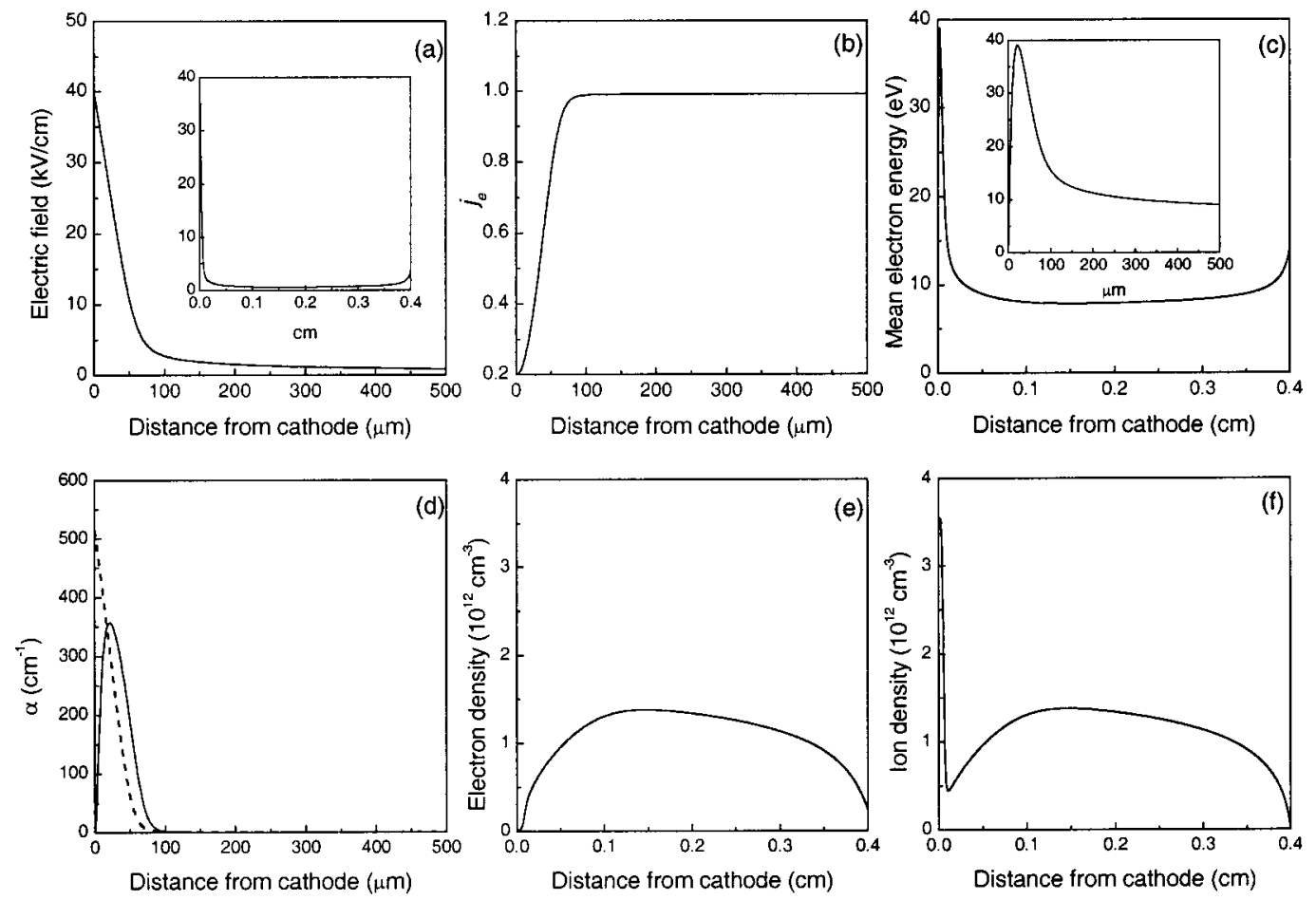

FIG. 2. Structural characteristics of (a) the electric field; (b) the normalized electron current density; (c) the mean electron energy; (d) the ionization coefficient estimated kinetically (solid curve) and hydrodynamically (dashed curve); (e) the electron density; and (f) the ion density in a helium dc APGD.

ization coefficient $\alpha$ in Fig. 2(d), both of which suggest rapid acceleration of secondary electrons in the cathode fall region. So the normalized electron current density increases rapidly and reaches unity around the near-anode boundary of the cathode fall region [Fig. 2(b)].

The electron acceleration process is a prerequisite for electrons to reach equilibrium with the local electric field (after exiting the cathode fall region) and to acquire sufficient energy for significant ionization. Figure 2(e) shows clearly the considerable increase of electron production after $z=10-20 \mu \mathrm{m}$ where the mean electron energy reaches around $20 \mathrm{eV}$ and $\alpha$ reaches $200 \mathrm{~cm}^{-1}$. This is also related to the sharp decrease of the ion density in the $z=10-20 \mu \mathrm{m}$ region in Fig. 2(f), which is caused by the rapid rise of electron current density there and hence a proportional reduction of the ion current density. The very large ion density in the $z<100 \mu \mathrm{m}$ region is an additional indication of the presence of the cathode fall.

It is also interesting to note that in the boundary region between the cathode fall and the positive column (60-80 $\mu \mathrm{m}$ in Fig. 2) the electric field is very small yet the electron energy is still large [see Figs. 2(a) and 2(c)]. This is a result of the considerable election acceleration that occurs in the cathode fall region. Therefore for this boundary region the ionization coefficient evaluated using the local electric field is much smaller than that evaluated using the electron energy as illustrated clearly in Fig. 2(d). In other words the hydrodynamic model underestimates the ionization coefficient near this boundary region. Further comparison simulations suggest that the underestimated ionization is particularly severe at low current densities $\left(J<10 \mathrm{~mA} / \mathrm{cm}^{2}\right)$. Interestingly, this underestimate of the hydrodynamic model is partly com- pensated by its overestimated ionization near the cathode surface such that in practice the hydrodynamic model is a fairly approximate method for dc atmospheric glow discharges, particularly for $J=0.01-0.1 \mathrm{~A} / \mathrm{cm}^{2}$. However the hybrid model offers greater physical insight and is necessary to accurately model ionization processes for both small ( $J$ $\left.<10 \mathrm{~mA} / \mathrm{cm}^{2}\right)$ and large current densities $\left(J>0.1 \mathrm{~A} / \mathrm{cm}^{2}\right)$.

Outside the cathode fall region the electric field has a persistent plateau in the $z=0.05-0.35 \mathrm{~cm}$ region [see the inset in Fig. 2(a)] before rising slightly towards the anode (e.g., the anode fall ${ }^{17}$ ). The low electric field in the plateau region is related to the large electron density there [Fig. 2(e)] that considerably enhances plasma conductivity in the positive column. This low field region coincides with a similar plateau of the small mean electron energy in Fig. 2(c) and that of the small ionization coefficient in Fig. 2(d). The low electric field is also responsible for the diminishing difference between the electron and ion densities in this region [see Figs. 2(e) and 2(f)]. In this region $(z=0.1-0.3 \mathrm{~cm})$ the electron impact ionization is very weak so it is a relatively inactive region. Hence the current conduction is entirely due to electron drift towards the anode. These are typical properties of the positive column in a glow discharge ${ }^{17}$ Near the anode the mean electron energy goes up slightly from the plateau in response to the anode fall.

The helium discharge in Fig. 2 has spatial structure very similar to that typical of low-pressure glow discharges. ${ }^{17}$ This close similarity not only confirms the experimental observations that atmospheric glow discharges can indeed be generated and sustained at $\mathrm{dc},{ }^{20-29}$ but also allows understanding of low-pressure glow discharges to be extended for application to atmospheric glow discharges. Despite of the 


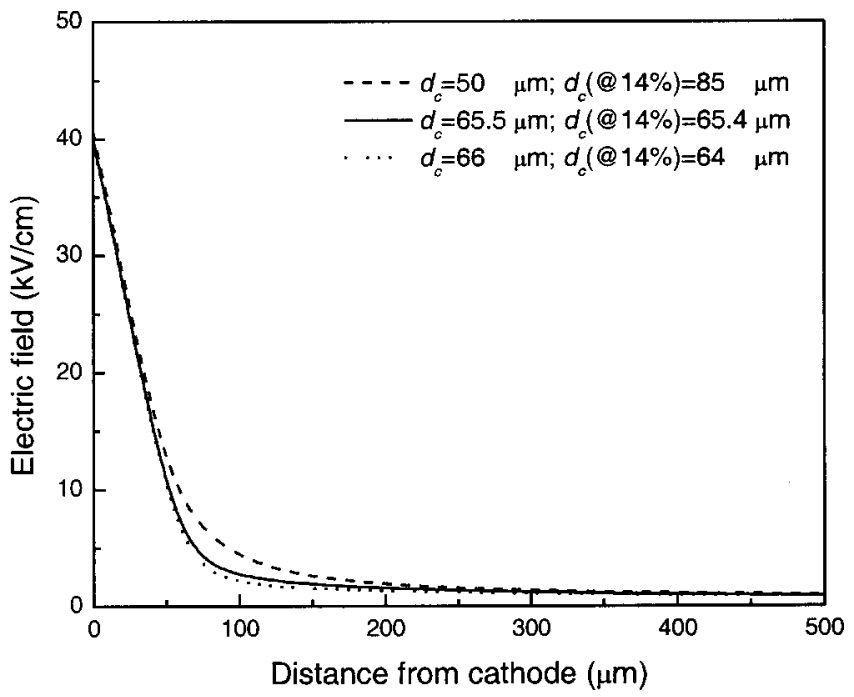

FIG. 3. Effects of different initial choices of cathode fall thickness on the electric field distribution.

simplicity of our model, it is capable of qualitatively reliable predictions, at least, of key physical quantities. For example, the mean electron energy is shown in Fig. 2(c) to be around $9 \mathrm{eV}$ in the space between the thin cathode fall layer and the anode, and this falls within the $1-10 \mathrm{eV}$ electron energy range estimated for APGD. ${ }^{1,2}$ The maximum electron density of $1.4 \times 10^{12} \mathrm{~cm}^{-3}$ in Fig. 2(e) and the maximum ion density of $3.5 \times 10^{12} \mathrm{~cm}^{-3}$ in Fig. 2(f) also broadly agree with experimental measurements ${ }^{2,14,15}$ and numerical modeling results. ${ }^{5,16,19}$ On the other hand, the total current density of $0.18 \mathrm{~A} / \mathrm{cm}^{2}$ needed to establish a cathode electric field of about $40 \mathrm{kV} / \mathrm{cm}$ is one magnitude lower than that measured in a rod-to-plane helium plasma experiment at atmospheric pressure. ${ }^{9}$ Much of the difference can be explained by the fact that Penning ionization and stepwise ionization ${ }^{7,16,19}$ are not included in our hybrid model. As a result, the hybrid model underestimates the actual ionization level and leads to a lower discharge current density. Hydrodynamic modeling suggests that the discharge current can be increased by a factor of 10 when Penning ionization and stepwise ionization are taken into account. ${ }^{7}$ Therefore the exclusion of Penning and stepwise ionization in our hybrid model is likely to be largely responsible for the difference between the numerically arrived at current density and the experimentally measured current density. In general, results of the simple hybrid model, summarized in Fig. 2, capture well the structure of dc APGD. The model can certainly be used both to understand basic features of dc APGD (e.g., cathode fall characteristics) and to explore their improvement, operating range, and possible scaling laws.

Several parameters are selected somewhat arbitrarily to initiate our numerical simulation. One such parameter is $d_{c}$, the thickness of the cathode fall region. Figure 3 shows the electric field profile at three different initial choices of $d_{c}$, and with value of $d_{c}$ determined numerically from the point at which the electric field is reduced to $14 \%$ of the cathode electric field. As is shown in Fig. 3, a too small an initial $d_{c}$, say, $50 \mu \mathrm{m}$, leads to slower decay of the electric field and to an overestimated $d_{c}$ from the $14 \%$ reduction rule, $85 \mu \mathrm{m}$ in this case. Similarly, if a larger initial $d_{c}$ is chosen, overestimation of the decay of the electric field results, leading to an underestimated $d_{c}$. In practice, the working equations are capable of achieving a single one-to-one correlation between an initial $d_{c}$ and its deduced value from the $14 \%$ rule. For the examples used in Fig. 2 this is $65.5 \mu \mathrm{m}$.

The results in Fig. 2 also rely on the similarly littledebated choice of $d_{1}=0.2 d_{c}$ and $\delta_{e}=0.5 \alpha$. Figure 4(a) shows that the electric field profile changes little when parameter $d_{1}$ is reset to $d_{1}=0.1 d_{c}$ and $0.3 d_{c}$, respectively, and the only clear change is the electric field in part of the plateau region between $z=100$ and $300 \mu \mathrm{m}$. This occurs outside the cathode fall and is unlikely to influence the discharge characteristics in a significant way. On the other hand, the production coefficient for excited species is assumed to be $\delta_{e}=0.5 \alpha$ since the ratio of cross sections for excitation and for ionization may be approximated by $0.5 .^{32,33}$ Although this approximation has been used successfully for low-pressure glow discharges, ${ }^{32,33}$ the actual ratio of the cross sections in fact decreases from 0.9 at $50 \mathrm{eV}$ to 0.4 at $200 \mathrm{eV},^{18}$ so it is useful to assess how this variation may impact the electric field profile. In Fig. 4(b) the electric field is plotted versus the distance from the cathode surface for $\delta_{e}=0.4,0.5$, and $0.7 \alpha$. Clearly there is very little impact on the field profile. Further computation of other parameters such as the mean electron energy and charged particle densities confirms that their changes due to difference in $d_{1}$ and $\delta_{e}$ are not significant and affect little the discharge structure in Fig. 2.

\section{RELATIONSHIPS AMONG KEY CATHODE FALL AND ELECTRICAL PARAMETERS}

Similar to the case of low-pressure glow discharges, we anticipate that generic relationships exist in APGD between key cathode fall parameters and other electrical parameters, for example, the cathode fall voltage, the cathode electric field, the current density, and the cathode fall thickness. These relationships offer valuable insight into the nature of generated glow discharges and their operating range, as well as allow useful comparison with low-pressure glow discharges.

Figure 5 shows the spatial profile of the electric field in and beyond the cathode fall region at different total current densities. It is clear that at large total current density the cathode electric field is greater. With a larger electric field secondary electrons acquire greater kinetic energy and produce more additional electrons through impact ionization. The combination of a larger electric field and a greater amount of additional electrons results in a larger total current density as suggested in Fig. 5. The broad proportionality between the electric field and the current density is characteristic of the ionization-dominant cathode fall region. On the other hand, the large cathode electric field also suggests a large ion density near the cathode surface and this requires the spatial derivative of the electric field to be larger according to the Poisson equation. This explains the faster reduction rate of the electric field at larger current density in Fig. 5. Consequently the electric field reduces to $14 \%$ of $E_{c}$ closer to the cathode surface leading to a narrower cathode 

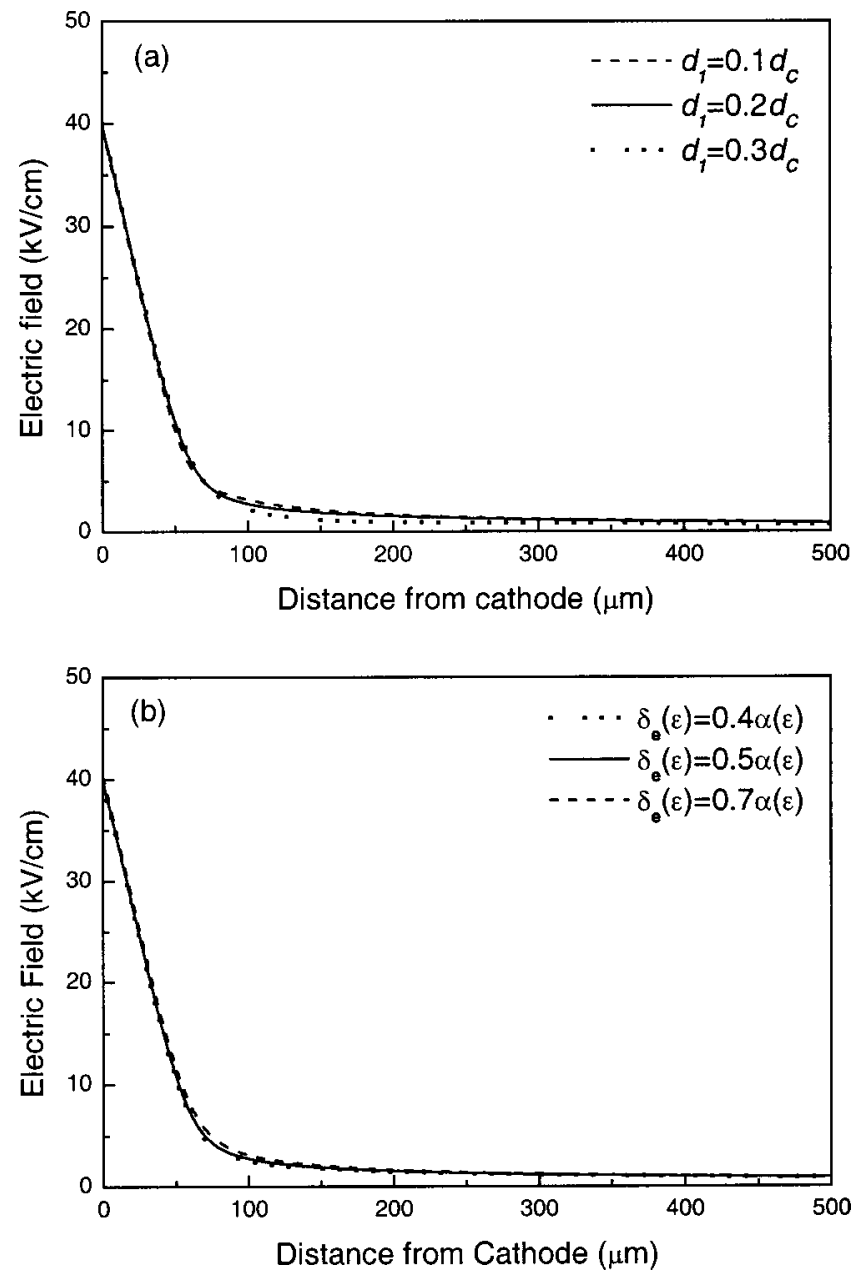

FIG. 4. Effects on the electric field distribution by (a) different $d_{1}$ and (b) different $\delta_{e}$.

fall region. This can be seen more clearly in Fig. 6 where the thickness of the cathode fall region is plotted as a function of the current density. It is broadly seen that $d_{c}$ is inversely proportional to $J$.

It is also of interest to compare the dependence of the cathode fall voltage, $V_{c}$, and the gap voltage, $V_{g}$, on the current density. $V_{c}$ is calculated by integrating the electric field across the cathode fall region of $d_{c}$ wide. In Fig. 7(a) the cathode fall voltage has a minimum of approximately $101 \mathrm{~V}$ when the total current is about $2 \mathrm{~mA} / \mathrm{cm}^{2}$. Given that the cathode fall region is dominated by ionization events, this graph highlights three important phases of a typical glow discharge, namely, the transition phase from the Townsend dark discharge to the normal glow discharge when the total current density is less than $1 \mathrm{~mA} / \mathrm{cm}^{2}$, the normal glow discharge phase of near-constant voltage ranging from $J$ $=1-4 \mathrm{~mA} / \mathrm{cm}^{2}$, and the abnormal glow discharge phase when the cathode voltage increases monotonically for $J$ $>4 \mathrm{~mA} / \mathrm{cm}^{2}$. The general trend of the $V_{c}-J$ relationship is very similar to that in low-pressure glow discharges, ${ }^{17}$ and the current density range of the normal glow phase agrees with that established in several numerical and experimental studies of kilohertz APGD in helium. ${ }^{7,34}$ In Fig. 7(b) the gap voltage is seen to decrease monotonically as the current den-

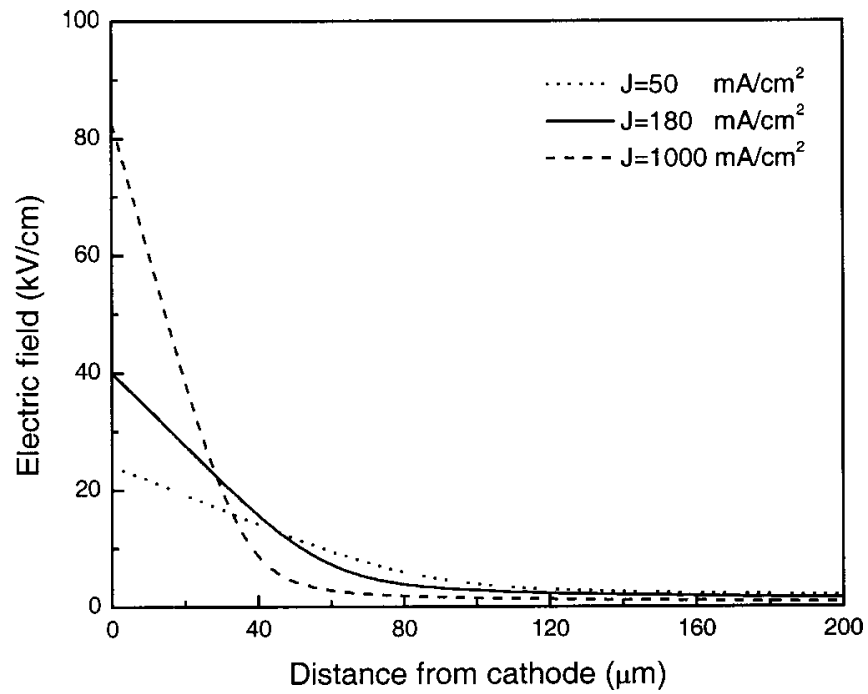

FIG. 5. Electric field distributions in the cathode fall region at different total current densities.

sity increases. A comparison between Figs. 7(a) and 7(b) suggests that the gap voltage is largely determined by the voltage in the positive column, $z=0.05-0.35 \mathrm{~cm}$ in our examples. Given that ionization is weak in the positive column, large current density causes more severe gas heating there hence the gas medium between the electrode gap becomes more conductive and thus reduces the gap voltage. ${ }^{17}$ The inverse proportionality between $V_{c}$ and $J$ is much more prominent at atmospheric pressure than at low pressures, as confirmed experimentally. ${ }^{10}$

Embedded in Figs. 6 and 7 is the relationship between the cathode fall voltage and the cathode fall thickness shown in Fig. 8. This may be understood as follows. Following initial gas breakdown between the two electrodes, the discharge structure is formed with a cathode fall region whose characteristics are largely determined by the voltage applied. If the voltage applied is increased further, there will eventually be subsequent breakdown. ${ }^{17}$ This occurs across the cath-

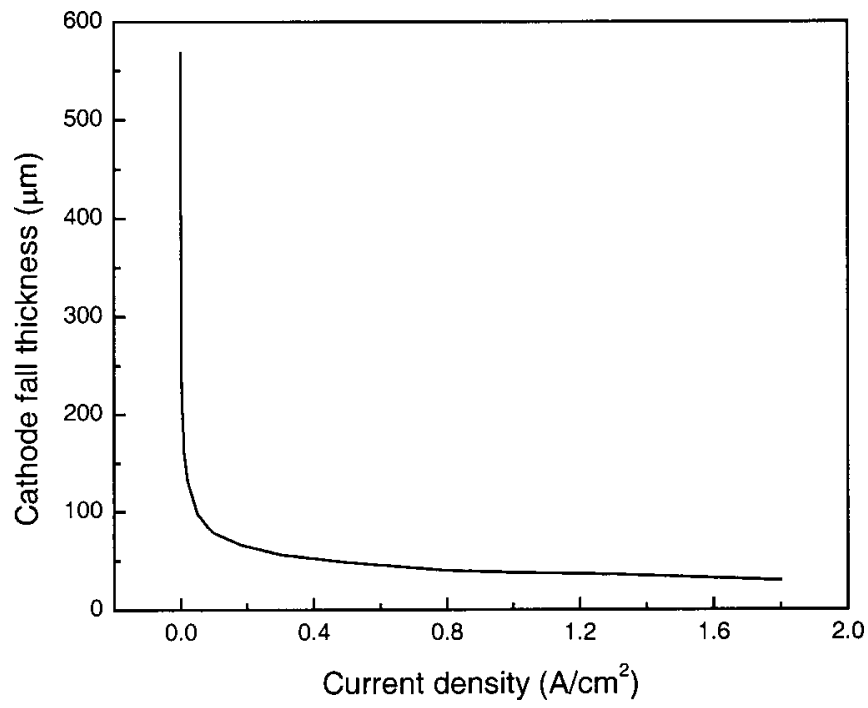

FIG. 6. Cathode fall thickness decrease as the current density increases. 

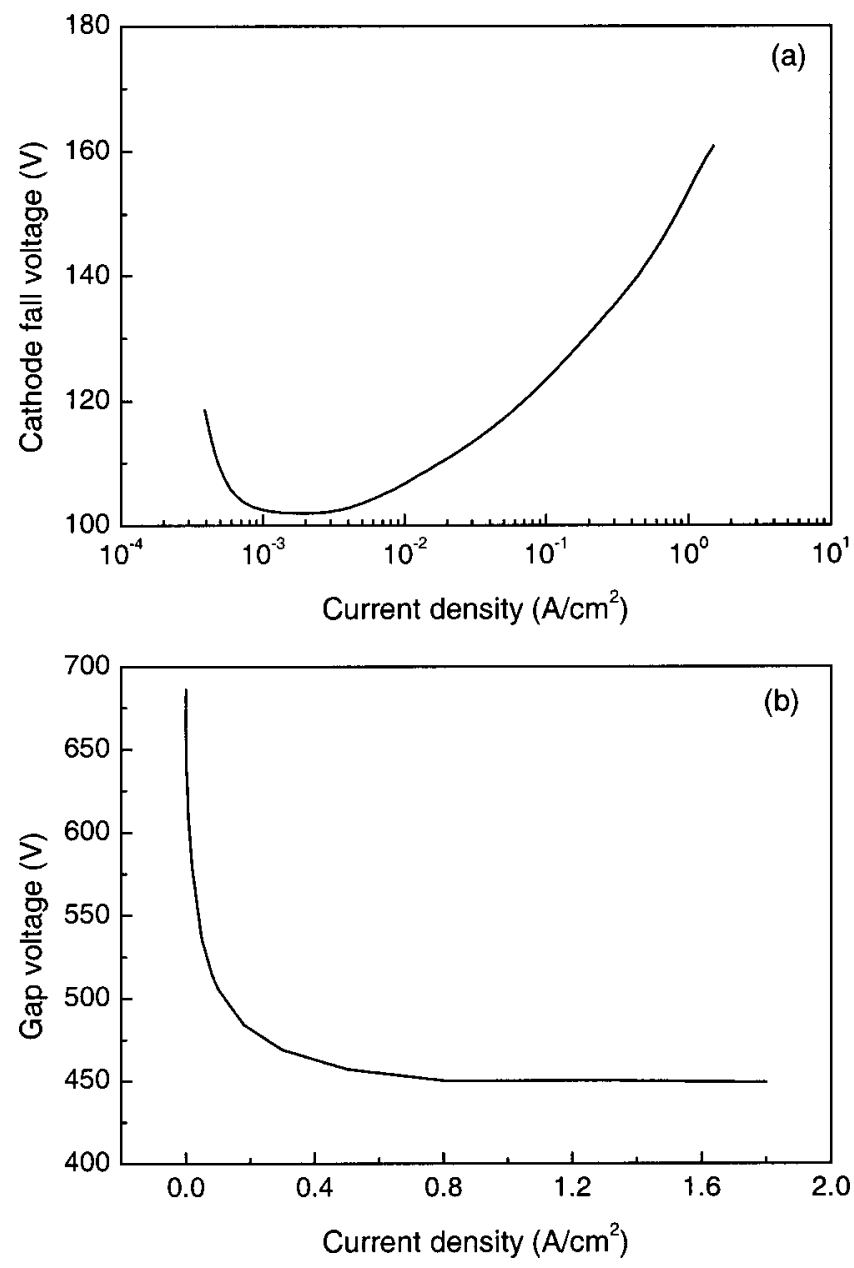

FIG. 7. Current density dependence of (a) the cathode fall voltage and (b) the gap voltage.

ode fall region where the electric field is much greater than that elsewhere in the interelectrode gap. Consequently, a new cathode fall region is formed, often with smaller thickness, in response to the larger applied voltage. The physical origin of this sheath breakdown is very similar to that of breakdown across a short gap in which no plasma is present initially. As shown in Fig. 8, the $V_{c}-p d_{c}$ relationship resembles the Pachen curve with $p d_{c}$ approximating $p d$. This supports the analogy between gas gap breakdown and sheath breakdown. At the voltage minimum, $p d_{c}=21$ (Torr $\mathrm{cm}$ ) is outside the 0.1-10 Torr $\mathrm{cm}$ range typically estimated for low-pressure glow discharges and but not dissimilar to the 10-20 Torr $\mathrm{cm}$ range established for a rf helium APGD experiment. ${ }^{35}$ The minimum breakdown field $E_{\mathrm{br}} \approx V_{c, \text { min }} / d_{c}=101 \mathrm{~V} / 0.028 \mathrm{~cm}$ $=3.6 \mathrm{kV} / \mathrm{cm}$ at $0.28 \mathrm{~mm}$ is, however, similar to the $3 \mathrm{kV} / \mathrm{cm}$ found experimentally for a $10 \mathrm{kHz}$ helium APGD. ${ }^{5}$

Although Fig. 5 establishes a qualitative correlation between the electric field and the current density in the cathode fall region, this is worth revisiting quantitatively. Figure 9 (a) shows a broad proportionality between the cathode electric field and the current density. As the current density increases over almost four orders of magnitudes from 0.4 to $2 \mathrm{~A} / \mathrm{cm}^{2}$, the cathode electric field increases also but only by a factor of 25 from 4 to $100 \mathrm{kV} / \mathrm{cm}$. Given that the current density is often proportional to the square of the electric field in low-

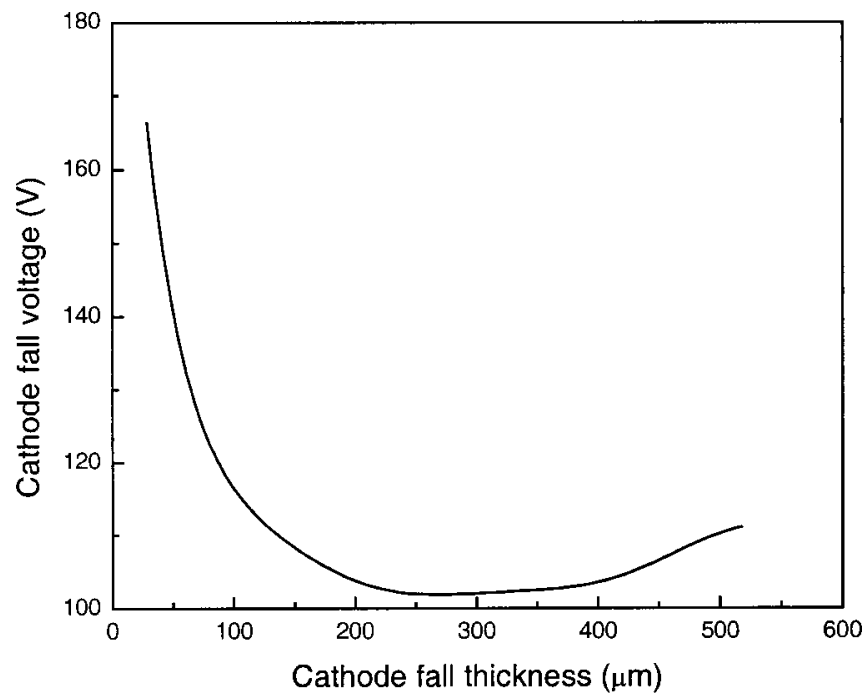

FIG. 8. Cathode fall voltage as a function of the cathode fall thickness.

pressure glow discharges, ${ }^{17,36}$ we plot $J / E_{c}^{2}$ as a function of the current density in Fig. 9(b) in which this ratio is seen to vary by a factor of 8 from 20 to $160 \mathrm{pA} / \mathrm{V}^{2}$. Numerical calculations suggest that $J / E_{c}^{2.5}$ varies much less, i.e., a factor of 1.5 from $J=0.4$ to $2 \mathrm{~A} / \mathrm{cm}^{2}$, than $J / E_{c}^{2}$. Thus at atmospheric pressure $J$ is approximately proportional to $E_{c}^{2.5}$, rather than to $E_{c}^{2}$. This is a distinct difference between dc APGDs and their low-pressure counterparts.

We now compare our numerical results with available experimental data in open literature that have sufficient detail about directly relevant quantities. Figure 10(a) shows the $J / E_{c}$ ratio plotted versus the current density for the cathode fall region, including our numerical results, data from an independent simulation, ${ }^{30}$ and data obtained in two dc APGD experiments. ${ }^{9,37}$ It is clear that the agreement is fairly favorable. Given that data for the cathode electric field are not available for many dc APGD experiments, we also consider the $J / E_{g}$ ratio for the entire interelectrode space with $E_{g}$ calculated as $V_{g} / L$, and this is plotted versus the current density in Fig. 10(b) in which data measured for three different experiments ${ }^{10,22,38}$ are also included. For the dc APGD experiment in Ref. 10, the cross-sectional area of the discharge column is not directly given so we estimate the effective diameter to be $93 \mathrm{~mm}$ from optical emission of the discharge column. Experiments in Ref. 22 employ platinum electrodes, whereas those in Refs. 10 and 38 use gold electrodes. The secondary emission coefficient due to $\mathrm{He}\left(2^{3} S\right)$ is known to be around 0.24 for platinum in helium, ${ }^{17}$ whereas there is little reliable data for $\gamma$ in the literature for gold in helium. Therefore we use $\gamma=0.25$ in our simulation. Compared to experimental data, the results of the hybrid model seem to overestimate the $J / E$ ratio by between $59 \%$ and $148 \%$. Again this is likely due to the exclusion of Penning and stepwise ionization in the hybrid model. With Penning and stepwise ionization taken into consideration, the cathode electric field will decrease for the production of the same amount of electrons (i.e., the same electron density). However these additional ionization mechanisms influence little the electric field in the positive column in which there 

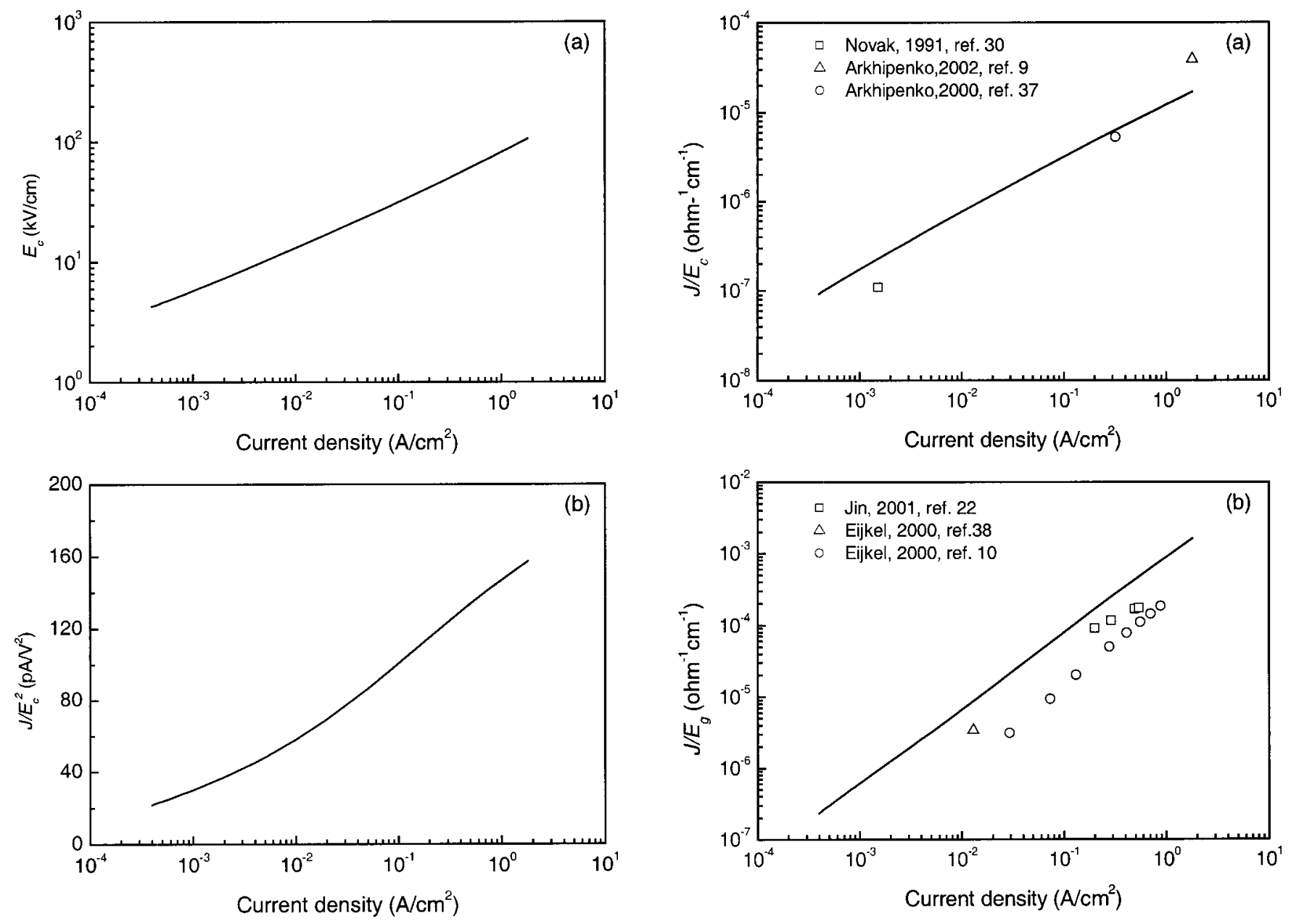

FIG. 9. Current density dependence of (a) the cathode electric field and (b) $J / E_{c}^{2}$.

are very few ionization events. The reduced electric field in the cathode fall region accelerates the electrons produced less than in the case without Penning and stepwise ionization, so the current density, $J=e n v$, is also smaller. To further accelerate electrons and compensate for the smaller current density, greater voltage in the positive column is needed. Given that the gap voltage is predominately determined by the voltage in the positive column (see Fig. 7), larger voltage in the positive column is likely to lead to a larger gap voltage even though the cathode fall voltage may be smaller. This will shift the numerical data (the solid curve) downward in Fig. 10(b) thus reducing their difference from experimental data. Therefore numerical predictions of the hybrid model compare well with experimental data and their remaining discrepancy is likely due to Penning and stepwise ionization not being included in the hybrid model.

In Sec. III it was mentioned that appropriate full kinetic modeling of APGD is currently not available to directly evaluate the proposed kinetic model of the cathode fall region [in Eq. (2)] and to assess the accuracy of approximating electron and ion transport parameters with their hydrodynamic values throughout the discharge region. We have established through Figs. 2 and 5-8 qualitative agreement of numerical predictions with relevant experimental observations and through Fig. 10 quantitative agreement with data of

FIG. 10. Current density dependence of (a) $J / E_{c}$ in the cathode fall region and (b) $J / E_{g}$ for the entire discharge region between the two electrodes. Solid curves are numerical results whereas markers are experimental data.

several dc APDG experiments. These favorable comparisons suggest that the hybrid model of Eqs. (10) and (11) are fundamentally sound and that hydrodynamic treatment of electron and ion transport parameters is a good approximation. Therefore the hybrid model can be applied to dc APGD reliably. Its advantage is simplicity, insightfulness, and wide applicability. As a result of our emphasis on simplicity and generic applicability, numerical results of the hybrid model are not meant to achieve quantitatively excellent agreement with experimental data. Nevertheless with additional features included, such as Penning and stepwise ionization in the case of helium discharges, the model can be expanded to provide quantitatively accurate predictions of experimental observations.

\section{CONCLUDING REMARKS}

A hybrid model was developed for dc excited atmospheric pressure glow discharges by kinetic treatment of their cathode fall region. Through extensive numerical examples, cathode fall characteristics of a helium dc APGD were unraveled and studied in detail. The spatial profiles of the electric field, the electron current density, the mean electron energy, and the electron and ion densities were shown to exhibit distinct structures of glow discharges that have a 
highly nonequilibrium cathode fall region and a nearly neutral positive column. These compare very well both with that in low-pressure glow discharges and with those measured electrically and spectroscopically for dc APGD. Several important relationships were also established, for example, that between the cathode electric field and the current density. Slightly different from the case of low-pressure glow discharges the current density in atmospheric glow discharges was found to be proportional to approximately $E_{c}^{2.5}$ rather than to the square of the cathode electric field. The dependence of the cathode fall voltage on the cathode fall thickness was found to be different to that of the gap voltage, yet both can be understood by basic glow discharge processes. Numerical prediction of the effective plasma conductivity, defined as the ratio of the current density over the electric field, was also compared to experimental data, and their agreement was found to be generally very good. These comparisons suggest that the hybrid model is fundamentally sound and that it can be applied reliably to dc atmospheric glow discharges. This simple model is particularly useful as a tool to gain basic understanding of the cathode fall characteristics in dc atmospheric glow discharges to establish their range of operation and possible scaling laws.

There is an interesting scope over which the hybrid model can be developed to offer greater accuracy and applicability. For example, it can be modified easily to include excited species and practically important impurities for helium APGD. ${ }^{5,7}$ These steps will allow the hybrid model to predict more accurately experimental results and to offer insight into the underlying plasma chemistry. Also, it is conceivable that by allowing time variation the hybrid model can be expanded to model ac excited atmospheric glow discharges and hence to improve results of hydrodynamic models that currently dominate the modeling of atmospheric glow discharges. These possible expansions and improvements will be reported in the future.

${ }^{1}$ A. Schutze, J. Y. Jeong, S. E. Babayan, J. Park, G. S. Selwyn, and R. F. Hicks, IEEE Trans. Plasma Sci. 26, 1685 (1998).

${ }^{2}$ E. E. Kunhardt, IEEE Trans. Plasma Sci. 28, 189 (2000).

${ }^{3}$ S. Kanazawa, M. Kogoma, T. Moriwaki, and S. Okazaki, J. Phys. D 21, 838 (1988).

${ }^{4}$ H. Koinuma, H. Ohkubo, T. Hashimoto, K. Inomata, T. Shiraishi, A. Miyanaga, and S. Hayashi, Appl. Phys. Lett. 60, 816 (1992).
${ }^{5}$ F. Massines, A. Rabehi, Ph. Decomps, R. B. Gadri, P. Segur, and C. Mayoux, J. Appl. Phys. 83, 2950 (1998).

${ }^{6}$ T. C. Montie, K. Kelly-Wintenberg, and J. R. Roth, IEEE Trans. Plasma Sci. 28, 41 (2000).

${ }^{7}$ M. G. Kong and X. T. Deng, IEEE Trans. Plasma Sci. 31, 7 (2003).

${ }^{8}$ Yu. S. Akishev, O. Goossens, T. Callebaut, C. Leys, A. Napartovich, and N. Trushkin, J. Phys. D 34, 2875 (2001).

${ }^{9}$ V. I. Arkhipenko, S. M. Zgirovskii, A. A. Kirillov, and L. V. Simonchick, Plasma Phys. Rep. 28, 858 (2002).

${ }^{10}$ J. C. T. Eijkel, H. Stoeri, and A. Manz, J. Anal. At. Spectrom. 15, 297 (2000).

${ }^{11}$ I. Alexeff and M. Laroussi, IEEE Trans. Plasma Sci. 30, 174 (2002).

${ }^{12}$ M. Laroussi, I. Alexeff, J. P. Richardson, and F. F. Dyer, IEEE Trans. Plasma Sci. 30, 158 (2002).

${ }^{13}$ M. J. Shenton and G. C. Stevens, IEEE Trans. Plasma Sci. 30, 184 (2002).

${ }^{14}$ A. P. Yalin, C. O. Laux, C. H. Kruger, and R. N. Zare, Plasma Sources Sci. Technol. 11, 248 (2002)

${ }^{15}$ S. E. Babayan, G. Ding, G. R. Nowling, X. Yang, and R. F. Hicks, Plasma Chem. Plasma Process. 22, 255 (2002)

${ }^{16}$ F. Tochikubo, T. Chiba, and T. Watanabe, Jpn. J. Appl. Phys., Part 1 38, 5244 (1999)

${ }^{17}$ Yu. P. Raizer, Gas Discharge Physics (Springer, Berlin, 1991).

${ }^{18}$ T. N. An, E. Marcode, and P. C. Johnson, J. Phys. D 10, 2317 (1977).

${ }^{19}$ X. H. Yuan and L. L. Raja, Appl. Phys. Lett. 81, 814 (2002).

${ }^{20}$ T. Cserfalvi and P. Mezei, Fresenius J. Anal. Chem. 335, 813 (1996).

${ }^{21}$ H. J. Kim, J. II. Lee, M. Y. Kim, T. Cserfalvi, and P. Mezei, Spectrochim. Acta, Part B 55, 823 (2000).

${ }^{22}$ Z. Jin et al., Anal. Chem. 73, 360 (2001).

${ }^{23}$ H. K. Jeong, S. C. Kim, C. Han, H. Lee, H. K. Song, and B. K. Na, Korean J. Chem. Eng. 18, 196 (2001).

${ }^{24}$ St. Kropke, Yu. S. Akishev, and A. Hollander, Surf. Coat. Technol. 142144, 512 (2001)

${ }^{25}$ R. K. Marcus and W. C. Davis, Anal. Chem. 73, 2903 (2001).

${ }^{26}$ O. Goossens, T. Callebaut, Yu. Akishev, A. Napartovich, N. Trushkin, and C. Leys, IEEE Trans. Plasma Sci. 30, 176 (2002).

${ }^{27}$ J. A. C. Brokaert, Anal. Bioanal. Chem. 374, 182 (2002).

${ }^{28}$ J. Chen and J. Davidson, Plasma Chem. Plasma Process. 22, 199 (2002).

${ }^{29}$ L. Yu, C. O. Laux, D. M. Packan, and C. H. Kruger, J. Appl. Phys. 91, 2678 (2002).

${ }^{30}$ J. P. Novak and R. Bartnikas, IEEE Trans. Plasma Sci. 19, 95 (1991).

${ }^{31}$ A. L. Ward, J. Appl. Phys. 33, 2789 (1962).

${ }^{32}$ A. J. Davies and J. G. Evans, J. Phys. D 13, L161 (1980).

${ }^{33}$ A. J. Davies, Proc. IEEE 133, 217 (1986).

${ }^{34}$ L. Mangolini, K. Orlov, U. Kortshagen, H. Heberlein, and U. Kogelschatz, Appl. Phys. Lett. 80, 1722 (2002).

${ }^{35}$ J. Park, I. Henins, H. W. Herrmann, and G. S. Selwyn, J. Appl. Phys. 89, 15 (2001).

${ }^{36}$ V. P. Nagorny, P. J. Drallos, and W. Williamson, Jr., J. Appl. Phys. 77, 3645 (1995)

${ }^{37}$ V. I. Arkhipenko, S. M. Zgirovskii, N. Konjevic, M. M. Kuraica, and L. V. Simonchik, J. Appl. Spectrosc. 67, 910 (2000).

${ }^{38}$ J. C. T. Eijkel, H. Stoeri, and A. Manz, Anal. Chem. 72, 2547 (2000). 